\title{
Beiträge zur Kenntnis spät- und postglazialer Akkumulation im nördlichen Alpenvorland ${ }^{1}$ )
}

\author{
Von Rüdiger Gernan und Paul Filzer, Tübingen
}

Mit 2 absoluten Altersbestimmungen des $C^{14}$-Laboratoriums Heidelberg und einem botanischen Beitrag von K. M. KHAN, Haiderabad (Pakistan) früher Tübingen.

\section{Mit 2 Abbildungen im Text}

$\mathrm{Z}$ u s a m me n f a s sung. Von mehreren Stellen des nördlichen Alpenvorlandes werden spätund postglaziale Profile aus einem periglazialen und einigen glazifluvialen Tälern beschrieben. Radiokarbonmethode und Pollenanalyse liefern die Zeitbestimmung für die Ablagerung der Sedimente. Auf diese Weise können mehrere Akkumulationsphasen im Spät- und Postglazial nachgewiesen werden. Sie liegen etwa in der Zeit der Stufen II, III und IV im Sinne von Brunnacker (1960, S. 86), bzw. in den Pollenzonen III, V und VIII. Außerdem sprechen mehrere Vorkommen für eine weitere Akkumulationszeit, ca. 6600 Jahre vor heute, in der Pollenzone VI. Diese jungen Aufschüttungen in unseren Tälern erreichen teilweise mehrere Meter Mächtigkeit. Sie sollten bei allen sich bietenden Gelegenheiten mit sämtlichen Hilfsmitteln der Quartärforschung untersucht werden, um ein möglichst genaues Bild der Flußarbeit und des Klimaablaufs im Holozän zu erhalten.

$\mathrm{Sum} \mathrm{mary}$. Late and postglazial profiles of the prealpine belt from periglacial and glacifluvial valleys are described. The time of sedimentation can be fixed by the radio carbon method and by pollen analysis. In this manner we can prove several accumulation phases in the Late- and Postglazial. They are found in stages II, III, and IV as defined by BRUNNACKER (1960, P. 86), and/or in the pollen zones III, V, and VIII. Some localities indicate a new accumulation phase about 6600 years B. P. in the pollen zone VI. These young accumulations in our valleys extend to several meters. For reconstruction of the river work and the history of the climate in the Holocene it is very important to examine the young valley sedimentation with all possibilities of quaternary sciences.

\section{In h a $1 \mathrm{t}$}

I. Einleitung (R. German) . . . . . . . . . . . . . . . 108

II. Das Profil der Baugrube Kläranlage Krumbach (R. German) . . . . . . . . . 109

III. Pollenanalytische Ergebnisse des Profils Krumbach (P. FILzer) . . . . . . 111

IV. Das Profil im Steingraben östlich Traunstein (R. German) . . . . . . . . 113

V. Überblick über die bisherigen Nachweise der Jüngeren Tundrenzeit im nördlichen Alpenvorland

a) Pollenanalytische Nachweise einiger Akkumulationsphasen des Spät- und Postglazials (P. Filzer) .

b) Geologisch-morphologischer Nachweis (R. German) . . . . . . . . 116

VI. Úberblick über die Akkumulationsphasen seit dem Spätglazial (R. German) . . . 118

VII. Literaturverzeichnis

\section{Einleitung (R. German)}

Die jahrzehntelang herrschende Lehrmeinung über glaziale Akkumulation und spätsowie postglaziale Erosion im nördlichen Alpenvorland wurde erstmals durch GrauL \& Groschopf (1952) erschüttert. Im vermeintlich hochglazialen Illerschwemmkegel bei Ulm (SChaefer 1940) wurden Baumstämme der Eiche, vorgeschichtliche Werkzeuge und Pollenkörner aufgefunden, welche zur Revision der Altersstellung des Illerschwemmkegels zwangen. K. Brunnacker (1960) und P. Groschopf (1961) haben diesen Problemkreis junger Akkumulation weiter verfolgt. Eine $\mathrm{C}^{14}$-Bestimmung "aus dem Wurzelansatz der Eiche“ im Illerschwemmkegel führte bei der Probe H 124/138 (Groschopf 1961) zu dem Wert

1) Geländeruntersuchung und Fertigstellung der Arbeit erfolgten mit Unterstützung der Deutschen Forschungsgemeinschaft. 
$1850 \pm 75$ Jahre. Wir haben aber nach den bisherigen Untersuchungen wahrscheinlich nicht nur diese eine Aufschüttungsperiode im Postglazial. BRUNNACKER (1960, S. 86) kam auf Grund seiner geologisch-bodenkundlichen Untersuchungen zu einer recht differenzierten Gliederung des spät- und postglazialen Klimaablaufes und konnte dabei 7 Stufen der spät- und postglazialen Akkumulation unterscheiden. Ähnlich möchte Groschopf (1961, S. 142) das Postglazial differenzieren und lehnt einen einzigen „Klimasturz“ ab.

Es erhebt sich daher die Frage: Welche Änderungen des postglazialen Klimaablaufes lassen sich geologisch nachweisen? Um nicht nur ganz lokale Verhältnisse zu erarbeiten, sollten sich diese Spuren auf längere Erstreckung, möglichst in den Ablagerungen von Flußläufen nachweisen lassen. Dies ist in den jungen Sedimenten oft nicht ganz einfach, da diese meist nur wenig über den Grundwasserspiegel herausragen und damit schwer zugänglich sind. Es handelt sich im vorliegenden Fall somit um die Untersuchung der jüngsten Flußaufschüttungen, bzw. der Ablagerungen im Hangenden der hoch- und spätglazialen Schichten. Diese Deckschichtenanalyse hat Soergel (1932) besonders im nichtvereisten Vorland häufig verwendet und Brunnacker (1952, 1960 u. a.) im Alpenvorland mit Erfolg eingeführt. Wie die Zusammenstellung durch H. Bremer (1960) zeigte, hat diese Methode in anderen Gebieten Deutschlands schon zu brauchbaren, wenn auch noch nicht immer einheitlichen Ergebnissen geführt.

Bei Untersuchungen im nördlichen Alpenvorland während des Sommers 1961 (durchgeführt mit Unterstützung durch die Deutsche Forschungsgemeinschaft) gelangte Verf. an verschiedenen Lokalitäten zu ähnlichen Ergebnissen wie Brunnacker (1960). Glücklicherweise konnten in fluviatilen Ablagerungen an 4 Stellen Holzreste aufgefunden werden. Damit entfiel für bestimmte Teile jener Schotterkörper bereits die früher angenommene kaltzeitliche Bildung. Es erhob sich aber sofort die Frage: In welche Zeit sind dann diese Bildungen einzureihen? In einem Vortragsreferat hat Verf. (GERMAN, im Druck) einige Ergebnisse dieser Geländeuntersuchungen festgehalten. Im folgenden sollen einige andere beweiskräftige Punkte dieses Sachgebietes herausgegriffen und dargestellt werden. Diese Ergebnisse besitzen deshalb einiges Interesse, weil eines der näher untersuchten Profile (Krumbach) aus einem Tal des periglazialen Vorlandes stammt und somit nicht vom Geschehen am Eisrand abhängig ist. Dadurch ergeben sich wichtige Vergleichsmöglichkeiten mit glazifluvialen Schmelzwasserrinnen.

\section{Das Profil der Baugrube Kläranlage Krumbach (R. German)}

In der Talaue der Kammlach (Iller-Lechplatte) wurde am nördlichen Ortsausgang von Krumbach (Schwaben) im Sommer 1961 eine Kläranlage in einer Meereshöhe von wenig über $500 \mathrm{~m}$ NN erstellt. Die Kammlach ist ein Flüßchen, welches im jüngeren Quartär keine glazifluvialen Schmelzwässer erhielt und somit zu den Gewässern des periglazialen Vorlandes zählt. Die Talaue der Kammlach ist bei Krumbach, am Mittellauf des Flüßchens, etwa 200-300 m breit. Die umgebenden Höhen erreichen kaum $600 \mathrm{~m}$ NN.

Zur Vorbereitung des Bauprojekts wurde eine Bohrung abgeteuft. Das von der Bauleitung ( $\mathrm{Fa}$. Baresel-Stuttgart) freundlicherweise zur Verfügung gestellte Profil zeigt (etwas vereinfacht):

$$
\begin{aligned}
& \text { In } 2 \mathrm{~m} \text { Tiefe Pflanzenreste } \\
& \text { bis } 3-3,5 \mathrm{~m} \text { Kies } \\
& \text { bis } 14 \text { m Sand mit etwas Kies } \\
& \text { (ab } 10 \text { m stellenweise Ton). }
\end{aligned}
$$

Beim Besuch der Baugrube konnte am 22. 6. 1961 an der Südwestecke folgendes Profil aufgenommen werden: 


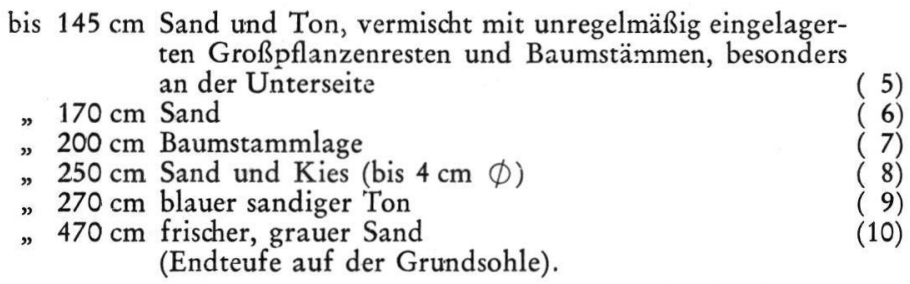

Die Lagen (5) und (7) deuten durch die Einlagerung der Holzreste auf Entstehung durch Hochwässer. Anscheinend floß die Kammlach schon bei der Aufschüttung der ganzen Serie im Osten der Baugrube, also etwa in der heutigen Gegend, da die Schichten alle nach Osten, gegen den Bach zu etwas abfallen.

Das Bohrprofil zeigt - unter Berücksichtigung des in Rechnung zu stellenden Nachfalls - wohl weitgehend die gleichen Verhältnisse wie das an der Baugrube aufgenommene Profil. Wir können daher die Angaben der Bohrung recht gut auswerten. Die Schicht (a) dürfte etwa den Schichten (1) bis (8), die Schicht (b) den liegenden Teilen (9) und (10) entsprechen. $\mathrm{Da}$ es sich bei den Schichten (9) und (10) der Baugrube sicher um keine anstehende Molasse handelt, dürfte die quartäre Talfüllung in der Gegend des unteren Endes der Bohrung, wenn nicht noch etwas tiefer, liegen. Gegenüber einigen anderen periglazialen Tälern, bei denen eine viel geringere Mächtigkeit der Talfüllung bekannt ist, erscheint die reiche Sedimentabfolge bei Krumbach auffallend.

Um Alter und Bildungsumstände dieser Aufschüttung einer periglazialen Rinne kennenzulernen, wurden folgende Proben für C ${ }^{14}$-Datierung und für Pollenanalyse entnommen:

Aus Schicht (3) die Probe 148

(4) 149

(5) 150 (vom unteren Teil)

(7) 120 (tiefster Teil der Schicht)

(8) 121 (H 1631-1197, Nr. Ge 1961/20)

(9) 151

Die Probe aus der Schicht (8) stellt das tiefste beobachtete Holz dar. Es lag bereits inmitten des Kieses etwa $20 \mathrm{~cm}$ unter der Baumstammlage (7). Das vorläufige Meßergebnis nach der Mitteilung von Herrn Phys. Ing. D. Berdau (II. Physikalisches Institut der Universität Heidelberg) vom 27. 7. 1962 lautet:

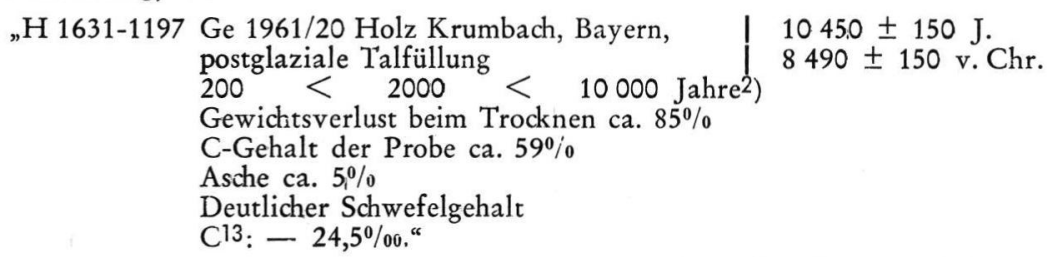

Dieses Meßergebnis bedeutet, daß das Holz aus dem Anfang der Jüngeren Tundrenzeit stammt. Leider war gerade das altersbestimmte Holz für botanische Bestimmungen so schlecht erhalten, daß keine nähere Angabe möglich war. Die Bestimmung des Großholzrestes verdanke ich den Bemühungen von Herrn Dr. K. M. KHAN, damals am Institut für spezielle Botanik der Universität Tübingen, heute Universität Haiderabad (Pakistan). Dabei ergab sich:

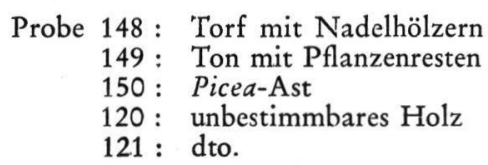

2) Bei Finsendung der Probe vermutetes Mindestalter, wahrscheinlichstes Alter bzw. Maximalalter. 
Mit dem Ergebnis, daß das altersbestimmte Holz in den älteren Teil der Jüngeren Tundrenzeit gehört, sind folgende weitere Aussagen möglich:

a) Nicht alle Baumstammlagen im nördlichen Alpenvorland sind gleich alt. Eine Alterseinstufung neuer Funde kann nicht schematisch auf Grund der Ergebnisse vom Illerschwemmkegel oder der vorliegenden erfolgen.

b) Die Aufschüttung der periglazialen Rinnen war keineswegs im Hochglazial beendet, sondern hat auch im Postglazial noch stattliche Beträge erreicht.

c) Die Sedimente des Spätglazials scheinen sich von denen des Hochglazials wie denen des Postglazials zu unterscheiden. Das läßt Schlüsse auf die Transportkraft des Wassers, wie auch auf die Zulieferung und Aufbereitung des transportierten Materials zu.

d) Die Jüngere Tundrenzeit ist in den Sedimenten eines Flüßchens nachweisbar. Durch die Altersbestimmung finden wir den Anschluß an die stratigraphische Deutung der Gliederung des Spätglazials und des früheren Postglazials bei BrunNacker (1960, Tab. 12).

\section{Pollenanalytische Ergebnisse des Profils Krumbach (P. Filzer)}

Unter den insgesamt $10 \mathrm{mir}$ von R. GERMaN zur pollenanalytischen Untersuchung übergebenen Proben lieferten zwei, aus Schicht (3) und (7), ein vollständiges, eine aus Schicht (10) ein rudimentäres Spektrum; die übrigen waren entweder pollenleer, oder es hatte eine so starke selektive Pollenzersetzung stattgefunden, daß die weitere Untersuchung nicht lohnte.

Schicht (3), vertreten durch Probe 148, lieferte folgendes Spektrum (Auszählung auf 200 Baumpollen): Kiefer 15,5\%, Fichte 26,5\%, Tanne 26,5\%, Rotbuche 21,5\%, Hainbuche $0,5 \%$, Eiche 3,0\%, Erle 5,0\%, Ahorn 1,0\%, Birke 0,5\%, Hasel 1,5\% und Kräuterpollen 7,0\% (darin 1,5\% Gräser, je 0,5\% Riedgras-, Compositen- und Caryophyllaceenpollen). Buche, Tanne und Hainbuche stellen also rd. 50\% des Baumpollens; der Eichenmischwald (EMW) ist dagegen nur mit 3,0\% der Eiche vertreten. Schicht (3) gehört also in das Subatlantikum (Nachwärmezeit); ob in dessen früheren, etwa nach 800 v. Chr. beginnenden und mit dem Einsetzen der mittelalterlichen Rodung endigenden Abschnitt (IX nach FIRBAS), wofür die schwache Vertretung der Nichtbaumpollen spricht, oder in Abschnitt X, läßt sich nicht sicher entscheiden; wahrscheinlicher ist das erstere.

Schicht (7) (Pr. 120/1). Das ebenfalls auf 200 Baumpollen basierende Spektrum lautet: Kiefer 32,5\%, Fichte 7,5\% ; Tanne 7,0\%, Buche 17,5\% ; Hainbuche 1,0\% ; Eiche 16,5\%; Linde 1,5\%; Ulme 0,5\%; Esche 0,5\%; Ahorn 1,0\% ; Erle 12,5\%; Birke 2,0\%; dazu Hasel 5,5\%; Kräuterpollen 11,0\% (darin 3\% Gras-, 2\% Riedgras-, 1\% Caryophyllaceen-, $0,5 \%$ Umbelliferenpollen). Gegenüber Schicht (3) ist also die Buche mit ihren beiden Begleitern nur mit ca. 25\%, der EMW dagegen mit 19\% vertreten; Schicht (7) läßt sich also pollenanalytisch der EMW-Buchenzeit (Subboreal, Späte Wärmezeit, Abschn. VIII nach FIRBAs) zuschreiben.

Wesentlich weniger ergiebig und auch sonst wenig befriedigend war das Polleninventar der Probe 147 aus der tiefsten Schicht (10). Nach der oben wiedergegebenen Cl4-Datierung des in Schicht (8) eingelagerten Holzes ist sie spätestens in die Jüngere Tundrenzeit (Abschn. III nach FIRBAS) zu stellen; höheres Alter ist jedoch stratigraphisch nicht auszuschließen.

In der mit $\mathrm{KOH}, \mathrm{HCl}, \mathrm{HF}$ und Azetolyse aufgeschlossenen Probe fanden sich beim Durchmustern mehrerer Präparate 6 Pollenkörner der Kiefer, 1 Lindenpollen und $1 \mathrm{zwei}$ felhafter Eichenpollen, ferner 9 Gras-, 1 Riedgras- und 4 Varia-Pollen. Außerdem traten aber auch mehrere Körner von Fichte, Tanne und Kiefer, z. T. als Fragmente auf, die sich in ihrer Beschaffenheit deutlich von den erstgenannten unterschieden: während die erst- 
genannten ziemlich transparent waren, waren die letzteren opak und entsprechen in ihrer Färbung dem üblichen Bild unangewitterten Pollens. Es liegt nahe, ihre Anwesenheit auf nachträgliche Einschwemmung eventuell während des Baues in die sehr lockere, sandigkiesige Schicht zurückzuführen. Sehen wir von ihnen ab, dann ergibt sich im Hinblick auf die Anwesenheit von fast ausschließlich Kiefernbaumpollen und der starken Beigabe von Kräuterpollen das - allerdings recht unscharfe - Bild einer spätglazialen Parktundra. Das Fehlen von Betula-Pollen und die warmzeitlichen Andeutungen machen eine Zuweisung zur Altesten Tundrenzeit $\left(I^{a}\right)$ sehr unwahrscheinlich; schon eher denkbar wäre die Zuweisung zur Älteren Tundrenzeit (Ic), obschon auch in diesem Fall ein sichtbarer Anteil der Birke zu erwarten gewesen wäre. Am ehesten paßt das rudimentäre Spektrum in die Jüngere Tundrenzeit (III); denn an der Schussenquelle (württ. Oberschwaben) fand G. LANG (1962) in einer Torfschicht, die er auf Grund einer Radiokarbondatierung der Jüngeren Tundrenzeit zuweist, einen fast reinen Kiefernwald mit zeitweise kräftiger Beigabe von Nichtbaumpollen. In diesem Falle wäre also nicht nur die durch Radiokarbonbestimmung wohl sicher der Jüngeren Tundrenzeit zugehörige Schicht (8), sondern auch Schicht (9) und (10) als Äquivalente der Jüngeren Tundrenzeit zu betrachten.

\section{Das Profil im Steingraben östlich Traunstein (R. German)}

Am Westrand des Salzachgletschers liegt dicht östlich Traunstein der Steingraben (Top. K. 1 : 25 000, Bl. 8142, Gitterzahl ca. 5302/4551). Er führt vom Moos „Pechschnait“ in WNW-Richtung am Außenrand eines Moränenwalles auf Traunstein zu. Bei dem Moränenwall handelt es sich offensichtlich um die Außere Jungendmoräne des Salzachgletschers. Dieser Vorlandgletscher besaß an seinem Westrand neben der Hauptzunge noch mehrere schmale, morphologisch weniger auffallende Nebengletscherzungen. Diese haben bisher offenbar verhindert, daß die Gesamtausdehnung richtig erkannt wurde. Darauf wird in anderem Zusammenhang näher eingegangen werden. Der Steingraben ist damit ein peripheres Schmelzwassertal der letzten Eiszeit.

Der Bach fließt aus dem Moos Pechschnait bei etwa $680 \mathrm{~m}$ NN heraus. Vom linken Talhang führen eine ganze Reihe von klingenartigen Tälern herunter. Die rechte Talseite zeigt dies nicht - mit Ausnahme der Kerbtäler beiderseits des Sporns bei Buchfalln, welcher wohl nicht mit letzteiszeitlichen Ablagerungen bedeckt ist. Dies ist wieder ein Hinweis, daß die periglaziale Abtragung, besonders die Runsenbildung (BüDEL 1960), im unvergletscherten Vorland des Salzachgletschers wirkte, während die Moränenablagerungen der Würmeiszeit davon verschont blieben. Talab folgt auf der rechten Seite ein Wäldchen, ehe sich der Bach des Steingrabens anschließend kräftig eingräbt. Auf Blatt 8141 der Top. Karte 1: 25000 führt die Schlucht den Namen Sparzer (Stein-) Graben.

Kurz ehe der Bach etwa zwischen der Einöde Buch und Obsölln im Süden an dem kleinen Wäldchen vorbeiführt, konnte an einem kleinen Prallhang auf der rechten Talseite folgendes Profil in etwa $640 \mathrm{~m}$ NN am 28. Juni 1961 aufgenommen werden:

$100 \mathrm{~cm}$ grauschwarzer Hangschutt

$0-10 \mathrm{~cm}$ obere Holzschicht (Probe 208/1)

$100 \mathrm{~cm}$ Kalktuff mit Schnecken und verkrusteten Zweigen

$0-10 \mathrm{~cm}$ untere Holzschicht (Probe 207/1)

$30-40 \mathrm{~cm}$ grobe Block- und Kieslage, Bachablagerung

Liegend sind $30 \mathrm{~cm}$ frische Grundmoräne bis zum Wasserspiegel des Baches aufgeschlossen.

Aus den Schichten (2) und (4) wurden die oben bezeichneten Proben für absolute Altersbestimmung entnommen. Bei den Holzresten handelt es sich nach der Bestimmung durch K. M. KHAN in beiden Fällen um Picea-Holz. Die absolute Altersbestimmung der Holzprobe aus der Schicht (2) über dem Kalktuff ergab laut obengenannter Mitteilung: 


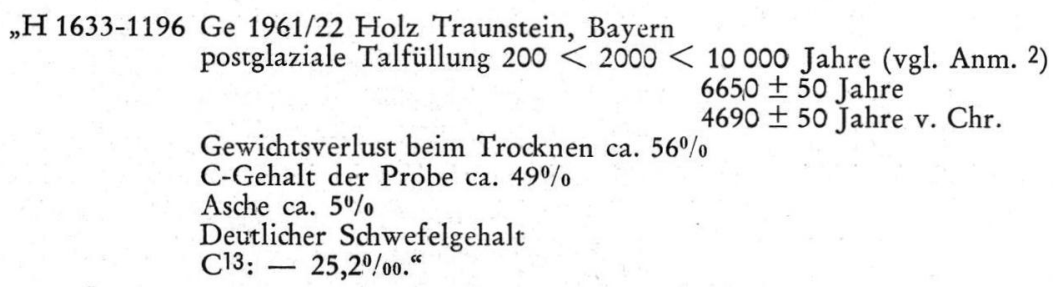

A u s we r t u ng. Damit gehört das Holz der oberen Schicht (2) in das Atlantikum. Der Kalktuff (3) könnte möglicherweise in die Frühe Wärmezeit gestellt werden, wie dies Groschopf (1961) für Kalktuffe Württembergs allgemein nachweist. Die etwas größere Meereshöhe vorstehend besprochener Bildung gegenüber den Objekten bei GroschopF (1961) und die Lage am Alpenrand einerseits und im Stufenland andererseits lassen eine etwas jüngere Einstufung und zwar in die Mittlere Wärmezeit nicht unmöglich erscheinen. Immerhin ist die Ưbereinstimmung bei Berücksichtigung der Lageunterschiede recht beachtlich. Auffallend ist, daß diese Hölzer, wie auch diejenigen von Krumbach, in einer feuchten bzw. maritimen Zeit gewachsen sind. Da beide Hölzer mit großer Wahrscheinlichkeit bald nach ihrer Bildung einsedimentiert wurden, hat wohl die damalige feuchte Zeit zu einer Überschwemmung der Talauen und Verschüttung der Hölzer geführt.

Auf diese Weise kennen wir zumindest 3 Phasen, welche seit der Allerödzeit zu Aufschüttungen (Überschwemmungen) in Talauen des nördlichen Alpenvorlandes geführt haben: vor ca. 2000 Jahren am Illerschwemmkegel, vor ca. 6500 Jahren bei Traunstein und vor ca. 10500 Jahren in der Jüngeren Tundrenzeit bei Krumbach.

\section{V. Überblick über die bisherigen Nachweise der Jüngeren Tundrenzeit im nördlichen Alpenvorland}

a) Pollenanalytische Nachweise einiger Akkumulationsphasen des Spät- und Postglazials (P. Filzer).

Daß der im Vorstehenden aufgezeigte Charakter der Jüngeren Tundrenzeit als einer Periode erhöhter geologischer Umlagerungstätigkeit nicht auf das engere Gebiet um Krumbach zu beschränken ist, lehrt ein Blick in das pollenanalytische Schrifttum. Wo immer im Alpenvorland ihr pollenanalytischer Nachweis geführt wurde [FIRBAS (1935) im Kolbermoor bei Rosenheim, LANG (1952) im Bodenseebecken, LANG (1962) an der Schussenquelle, Gronbach (1961) im Federseebecken, Götтlich (1962) im Hoßkircher Weiherried, Welten $(1944,1947)$ im Solothurnisch-bernerischen Mittelland und im Berner Oberland, v. SARnthein $(1940,1948)$ in Nordtirol], zeichnen sich die einschlägigen Schichten durch erhöhte Tonbeimengung aus. Das Einsetzen und Wiederaussetzen dieser Toneinschwemmung kann unmittelbar zur Abgrenzung der Jüngeren Tundrenzeit gegen die vorausgehende Alleröd- und die nachfolgende präboreale Kiefern-Birkenzeit verwendet werden.

Daß sich spätere Akkumulationsphasen im Alpenvorland bisher nur sehr viel weniger deutlich abzeichnen, dürfte in erster Linie darauf zurückzuführen sein, daß das in der Zwischenzeit erfolgte Vordringen des Waldes auch in höheren Gebirgslagen einen erhöhten Schutz gegen die Bodenabschwemmung schuf, so daß die im Vorland zur Ablagerung kommenden Materialmengen beträchtlich zurückgingen. Es wäre jedoch zu erwarten, daß unmittelbar am Alpenrand sich auch noch spätere Akkumulationsphasen abzeichneten. Daß dies wirklich der Fall ist, ergibt sich aus folgendem:

Zur Klärung der Altersstellung prähistorischer Funde im Gebiet des ehemaligen Lechsees bei Füssen hatte ReInenth im Jahre 1962 einige Profile erbohrt und mir zur pollenanalytischen Bearbeitung zur Verfügung gestellt. Die gesamten Ergebnisse dieser Untersuchung sind einer späteren Publikation vorbehalten. Da jedoch die untersuchten Profile 
für die Fragestellung German's einiges zu sagen haben, sei hier in Kürze auf die einschlägigen Abschnitte der Profile eingegangen. Es handelt sich zunächst um eine im Gebiet des Bannwaldsees bei Füssen (Buching-Berghof, Rotsarimoos II) entnommene Probeserie, die bis auf die in $10 \mathrm{~m}$ Tiefe anstehenden geschlossenen Tonschichten hinabreicht. In die hangende Seekreide ist nun in zweimaligem Wechsel je eine stark tonige Schicht eingelagert, die untere, ca. $15 \mathrm{~cm}$ mächtige bei $840 \mathrm{~cm}$, die obere, nur ca. $5 \mathrm{~cm}$ mächtige bei $770 \mathrm{~cm}$. Ihre pollenanalytische Altersstellung ergibt sich aus dem hier in seinen einschlägigen Teilen wiedergegebenen Diagrammausschnitt.

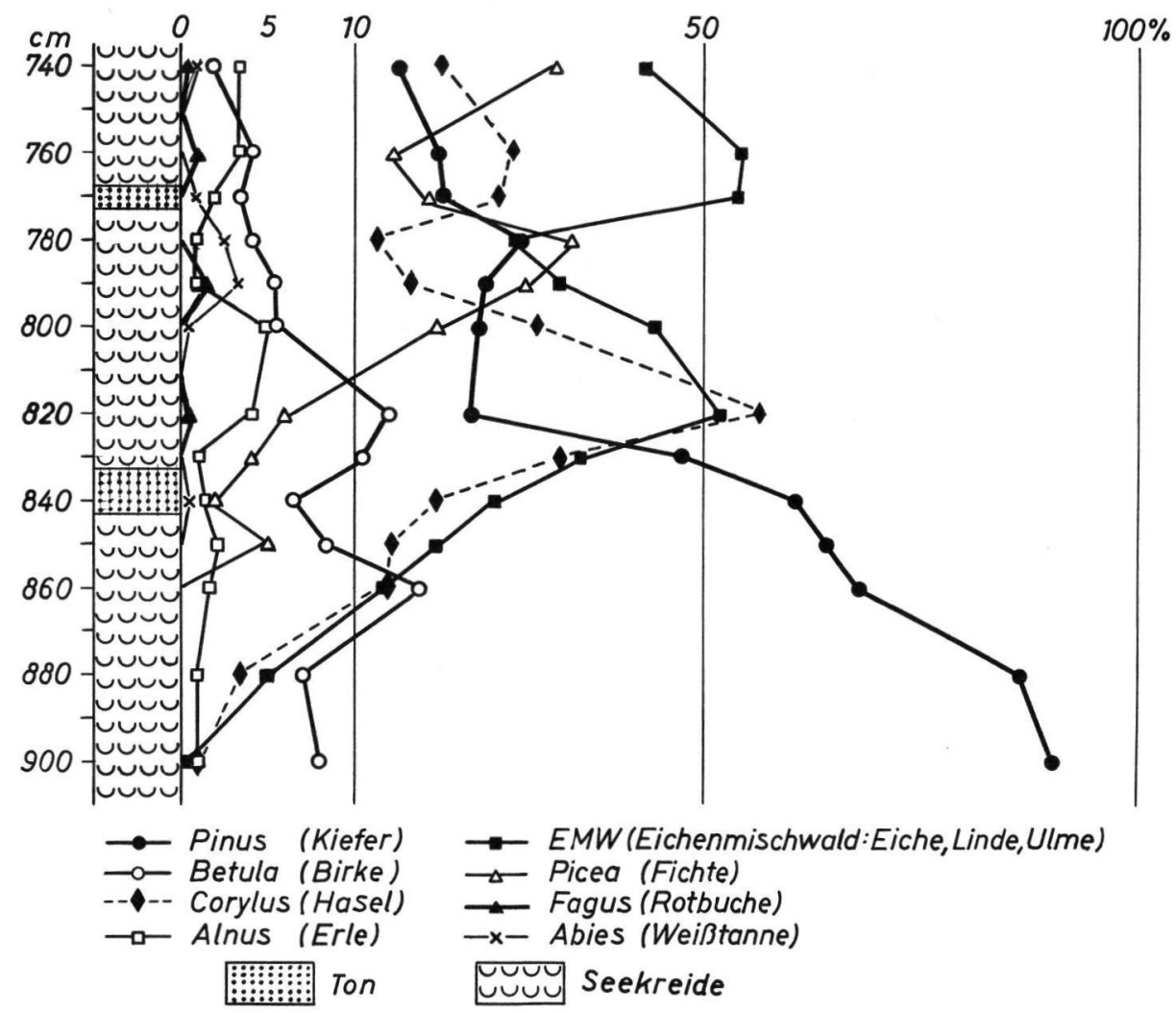

Abb. 1. Ausschnitt aus dem Pollendiagramm Rotsarimoos II bei Buching Kr. Füssen (Top. K. 1 : 25000 Bl. Roßhaupten, Nr. 8330). Am linken Rand ist die Tiefe des erbohrten Profils in Zentimetern unter der Oberfläche angegeben. Daneben folgt nach rechts die Sedimentart und das Pollenspektrum.

Die untere Tonschicht wurde demzufolge an der Wende von der präborealen KiefernBirkenzeit zur borealen Haselzeit etwa in der Mitte des raschen Anstiegs der Hasel- und der EMW-Kurve, die obere im Anschluß an eine Depression dieser beiden Kurven kurz vor der Erreichung des Hauptmaximums des EMW und eines synchronen zweiten, kleineren Haselgipfels abgelagert. Diese beiden Tonlagen sind offenbar der Ausdruck zweier postglazialer Phasen mit gesteigerter Umlagerung, die erste am Ende der Vorwärmezeit, also etwa ums Jahr 6800 v. Chr., die andere im ersten Teil der Hauptwärmezeit (Atlantikum), also wohl im 5. Jahrtausend v. Chr. oder noch etwas früher.

Betrachten wir zunächst die untere der beiden Tonzwischenlagen noch näher. Ihr pollenanalytischer Charakter zeichnet sich im Profil des Bannwaldsees nur wenig deutlich 
ab, ein kurzes „Auf-der-Stelle-Treten“ der Haselkurve, verbunden mit einem ersten, noch schwachen Vorstoß der Fichte. Sehr viel deutlicher tritt die Reaktion der Vegetation in zwei weiteren Profilen vom benachbarten Hopfensee (Hopfen-Halbinsel) hervor. In beiden zeichnet sich in den entsprechenden, ebenfalls durch Toneinlagerung ausgezeichneten Schichten folgendes ab: eine kurzdauernde, aber sehr deutliche Rückläufigkeit der im Anstieg begriffenen Haselkurve, anschließend eine schwächere der EMW-Kurve, damit verbunden ein deutlicher, ebenfalls kurzdauernder Fichtengipfel und eine vorübergehende Andeutung von Buche und Tanne.

Das gleiche Pollenbild weist nun ein weiteres Profil der Umgebung von Füssen (Schraden I) auf, nur daß sich hier die Phase erhöhter Akkumulation nicht in einer Tonanreicherung im Sediment manifestiert. Das läßt sich aber darauf zurückführen, daß die Untersuchungsstelle in größerer Entfernung von den Randbergen des Füssener Beckens liegt und im Zusammenhang damit der See hier schon lange vor der fraglichen Zeit das Spätstadium der Lebermudde, bzw. des Seggentorfs erreicht hatte.

Ein Studium der von PAUL \& RuOFF (1932) veröffentlichten Pollendiagramme aus dem Gebiet des ehemaligen Iller- und Lechgletschers lehrt nun, daß es sich bei dem von uns angetroffenen offenbaren Klimarückschlag nicht um eine auf das Gebiet von Füssen beschränkte Erscheinung handelt. Das am Fuße des Grünten, also ebenfalls in Alpenrandnähe liegende Gallmoos bei Agathazell (PAuL \& Ruoff, 1932, Abb. 89, S. 170) zeigt in pollenzeitlicher Koinzidenz eine in Waldtorf eingelagerte untere Tonschicht (über höhere Schichten s. u.). In größerer Entfernung vom Alpenfuß setzt sich in einigen weiteren Profilen zwar nicht das petrographische, aber doch das waldgeschichtliche Zeugnis dieser Klimaschwankung durch: Im Profil des Schornmooses bei Oberthingau (PAuL \& RuofF, Abb. 82, S. 15) steigt die Hasel in $330 \mathrm{~cm}$ zunächst auf ca. $35 \%$ an, fällt dann in $300 \mathrm{~cm}$ auf $14 \%$ zurück und erreicht in $270 \mathrm{~cm}$ ihr Maximum mit $90 \%$. Entsprechendes bietet das Diagramm II des benachbarten Unteren Brandholzmooses (PAUL \& RUOFF, Abb. 84, S. 163), und ähnliches findet sich in den weiter östlich gelegenen Geltnachmooren (PAUL \& Ruoff, Abb. 79, S. 153). Daß in anderen Diagrammen Äquivalente fehlen, dürfte teilweise dem zu großen Probenabstand zuzuschreiben sein; in niedrigen Meereshöhen mag sich die Klimaschwankung auch nur in geringerem Maße ausgewirkt haben. Firbas (1949) bemerkt hierzu folgendes: „Nur selten ist in den Diagrammen schon das erste CorylusMaximum zweigipfelig, z. B. im Hohen Venn (Michelshütte) und bei Urbis und im Maxmoor in den Vogesen. Die Bedeutung dieser Erscheinung, die anderwärts, z. B. in Dänemark und Südwestnorwegen, nicht selten ist, ist noch unbekannt. "Wir glauben, daß das engere Alpenrandgebiet den Schlüssel zum Verständnis dieser Erscheinung liefert; doch soll hierauf erst in einer späteren Veröffentlichung näher eingegangen werden.

Was nun die obere, nur geringmächtige Tonzwischenlage des Bannwaldseeprofils bei $770 \mathrm{~cm}$ betrifft, so zeichnet sich ihre pollenanalytische Signatur (Eindellung der im ganzen rückläufigen Hasel- und EMW-Kurve bei gleichzeitigem kräftigem zweiten Vorstoß der Fichte und einem zweiten, deutlicheren Hinweis auf die Anwesenheit bzw. Nähe von Buche und Tanne) auch in den Profilen von Schraden I und Hopfen-Halbinsel ab. Im Gallmoos am Grünten dürfte mit ihr eine zweite Tonlage in $340-350 \mathrm{~cm}$ Tiefe korrespondieren; möglicherweise gehört aber auch die dritte, besonders kräftige Tonschicht in $280-240 \mathrm{~cm}$ Tiefe hierher; eine klare Entscheidung ist infolge der in dieser Schicht festgestellten Pollenzerstörung nicht möglich.

Es ist naheliegend und wurde vorher auch schon angedeutet, daß die beiden hier herausgearbeiteten Vegetationsschwankungen das Werk eines Temperaturrückschlages sind. $\mathrm{Ob}$ damit gleichzeitig eine Erhöhung der Niederschläge verbunden war, ist schwer zu sagen; doch wird man in jedem Fall annehmen dürfen, daß auch bei gleichbleibendem Niederschlage eine Herabsetzung der Temperatur zu einer Verminderung der Verdunstung und damit zu einer Steigerung der Erosionsleistung der fließenden Gewässer führen konnte. 
Die letzte und im Hinblick auf das von German beigebrachte Material wichtige Frage, inwieweit sich die beiden soeben diskutierten Phasen gesteigerter Erosion und Akkumulation auch in erhöhter Tätigkeit der auf das periglaziale Vorland beschränkten Flüsse auswirken konnten, kann erst durch weitere Untersuchungen beantwortet werden.

b) Geologisch-morphologischer Nachweis (R. German)

In zwei Studien versuchte C. Rathjens (1952 und 1953) mit Hilfe von geomorphologischen Gesichtspunkten die Erscheinungen der JTZ ${ }^{3}$ ) zu fassen. Im Alpenvorland ist die JTZ nach LANG (1952) nicht durch eine Tundrenvegetation, sondern durch einen, wenn auch lichten Wald vertreten. Das bedeutet aber, daß während der JTZ im Alpenvorland der Boden durch Baumwurzeln zusammengehalten wurde. Abgesehen von jüngster anthropogener Bodenerosion dürfte die geomorphologische Formbarkeit des Alpenvorlandes in der JTZZ nicht wesentlich anders als heute gewesen sein. Die Existenz des Waldes dürfte eine Bodengefrornis von mehreren Zehnern von Metern Tiefe wenig wahrscheinlich machen, wie sie zur Herausarbeitung der vermeintlichen Formen notwendig wäre. Unter den nunmehr bekannten Gesichtspunkten wäre das Wasser in den Schottern der Niederterrassen versickert, ehe es hätte erodieren können. Die von RathJENs (1952) beschriebenen Formen in der Niederterrasse müssen daher älter sein.

Anzeichen nennenswerter geologischer Arbeit sind nach der Wiederbewaldung am Ende der Würm-Eiszeit danach nur an Flußläufen zu erwarten, wie dies hier an einigen Beispielen gezeigt wird. Nur an Flüssen wird durch die umgestaltende Wirkung des Wassers noch eine bemerkbare geologische Umarbeitung möglich sein.

In welche Zeit sind dann die „asymmetrischen Täler in den Niederterrassen des nördlichen Alpenvorlandes“ zu stellen und wie wurden sie gebildet? - Hinweise für die Beantwortung dieser Frage geben die oberen Enden der asymmetrischen Tälchen der Münchner Ebene.

Diese Tälchen beginnen dort keineswegs alle inmitten der Niederterrasse (RATHJENS, 1952, Abb. 1), sondern setzen sich talauf weiter fort (s. Abb. 2).

Am Beispiel des Trockentals östlich und südöstlich von Planegg läßt sich einwandfrei zeigen, daß in der Gegend der Höhenlinie 850 nördlich Buchendorf-Anfers keine Mulde eines asymmetrischen Tales liegt, sondern daß sich das Trockental nach Süden in die Altmoränenlandschaft fortsetzt. Im Gebiet der Altmoränenlandschaft läuft auf das asymmetrische Trockental ein ganzes Bündel periglazialer Trockentäler zu (vgl. Abb. 2). Diese Verlängerung des Trockentales beweist noch keineswegs ein anderes Alter als von RATHJENS (1952) angenommen. Entscheidend ist, daß in das Gautinger Trockental neben den Periglazialtälern außerdem auch noch Schmelzwasserrinnen einmünden, welche von der Moräne des würmeiszeitlichen Maximalstandes herführen, aber etwas jüngeren Stadien zugehören. Bei diesen besteht keinerlei Zweifel, daß sie hochglaziale Bildungen der Würmzeit sind. Wir müssen daher annehmen, daß sich folgende Ereignisse im Hochglazial kurz hintereinander abspielten: Ablagerung der Schotter auf der Münchner Ebene, Aufschüttung der Äußeren Jungendmoräne und erste Zerschneidung der Schotterdecke. Gleichzeitig mit diesen Erscheinungen und wahrscheinlich auch noch etwas später dürfte die periglaziale Abtragung der Altmoränenlandschaft erfolgt sein. Die Eintiefung der reinen Periglazialtäler und, wie RAthJENS mit Recht betont, der Schwemmkegel, die auf die Gautinger Stufe aufgelagert sind, deuten darauf hin, daß die periglaziale Abtragung möglicherweise noch etwas andauerte.

Am Südrand der Münchner Ebene gibt es aber auch eine ganze Reihe von Schmelzwasserrinnen, welche talab morphologisch keine Fortsetzung auf dem Schotterfeld zeigen, deren Flüsse offensichtlich versiegen. Bemerkenswerterweise liegt in deren Nähe oder kurz unterhalb jeweils mindestens ein asymmetrisches Tal. Es scheint daher, als ob im späten

3) JTZ = Jüngere Tundrenzeit. 


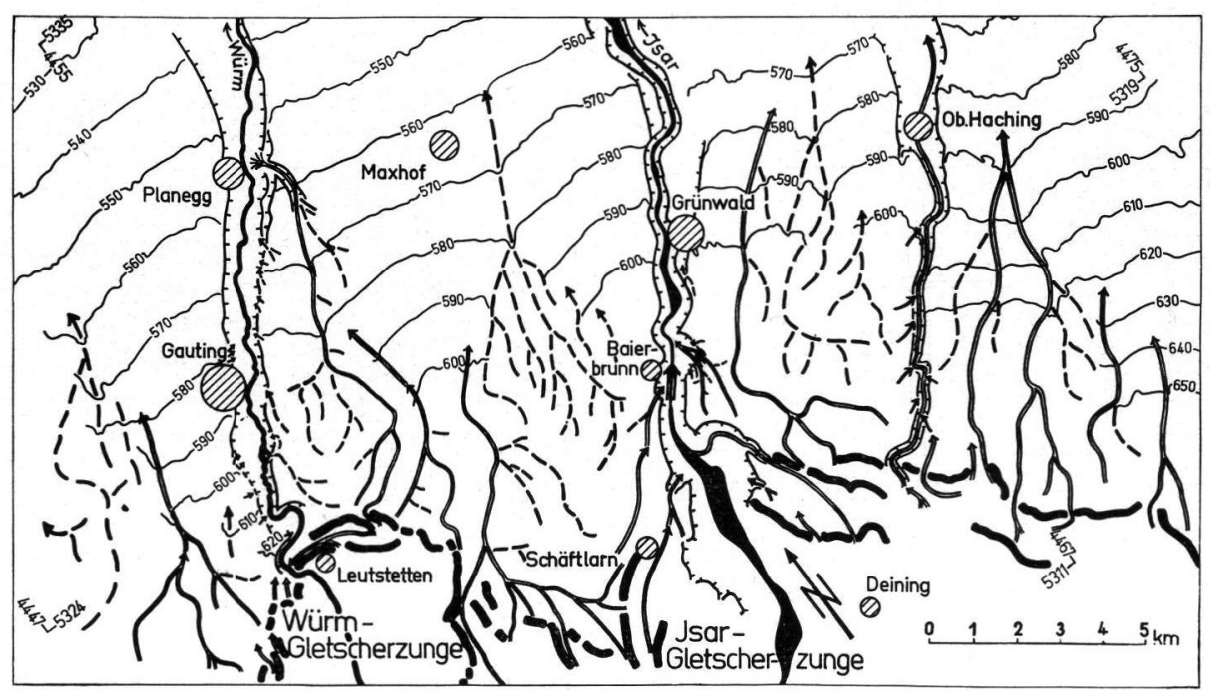

Abb. 2. Ausschnitt vom Südrand der Münchner Ebene. - Die Karte zeigt folgende Elemente: a) Di e w ürmzeitlichen W a $11 \mathrm{mor}$ än en bei Leutstetten-Schäftlarn (breit gezeichnete Linien). b) $\mathrm{Die} g \mathrm{großen} S$ anderflächen der einzelnen Eiszungen (mit ausgezogenen Höhenlinien im Abstand von 10 Metern gezeichnet, Höhe über NN). c) In seln d e r A $1 \mathrm{t}-$ mor änenlandschaft (ohne Höhenlinien gezeichnet) zwischen den Endmoränen und der Sanderfläche. Das periglaziale Talnetz dieser Gebiete ist gerissen gezeichnet (- - -). Rinnen, welche vermutlich schon vor der Würmeiszeit angelegt waren, sind zur Ausgestaltung eines periglazialen Talnetzes verwendet worden. Dieses Talnetz ist sehr dicht, solange es in der Altmoränenlandschaft verläuft. Auf der Niederterrasse vereinigen sich die einzelnen Zweige des periglazialen Talnetzes zu einer Hauptrinne. - Einige Täler wurden beim Maximalstand des Eises durch Akkumulation von Schottern vor der Wallendmoräne der Würmeiszeit in den glazifluvialen Bereich einbezogen. Meist wurde dadurch der Tallauf unbedeutend verlängert, indem ein neuer Oberlauf angesetzt wurde. Diese glazifluvialen Rinnen waren meist nur beim eigentlichen Maximalstand des Eises in Tätigkeit. Danach dienten die Rinnen - wie zuvor - zur Entwässerung des periglazialen Vorlandes. d) Glazifluviale $R$ innen des Maximalstandes sind in die Altmoränenlandschaft eingeschnitten und verbinden die Gletschertore in den Wallmoränen des Maximalstandes mit den Sanderflächen. Glazifluviale Rinnen sind mit doppelten Linien eingezeichnet. e) Gl a z if $1 \mathrm{u}$ vi a le $\mathrm{R}$ in n en der s pätere n Phasen. Einzelne Gletschertore mit besonders tiefer Lage dienten zur Entwässerung der beckeneinwärts liegenden Stadien. Sie durchziehen meist als tiefe Erosionskerben die Niederterrassenflächen. Diese Hauptschmelzwasserrinnen aus der Abschmelzzeit des Eises (Würm-, Isar- und Oberhachingertal) werden von Erosionskanten begleitet. Diente eine Rinne sowohl glazienuvialen, wie auch periglazialen Wassermassen zum Abfluß (z. B. östlich Planegg), so wurde aus Gründen einfacher Darstellung nur die glazifluviale Signatur eingezeichnet. f) K e r b e n in der Niederterrasse (s. Text).

Hochglazial, bzw. im frühen Spätglazial, d.h. kurz nach dem Maximalstand, viele Schmelzwasserströme in den weitflächigen Schottern der Münchner Ebene mehr oder weniger versiegten. Kurz talab können die Wassermassen jedoch in einer periglazialen Rinne, welche infolge ihrer tieferen Lage den Grundwasserstrom an sich zog, wieder zum Vorschein kommen. Wahrscheinlich bildeten gerade diese Grundwasserströme die Ursache für die besonders kräftige Ausbildung mancher dieser asymmetrischen Tälchen (vgl. Abb. 2 sïdwestlich und südöstlich Maxhof und westlich von Gauting).

Die Entstehung der asymmetrischen Täler in der Niederterrasse dürfte damit auf einer kombinierten Wirkung von periglazialen Entwässerungsrinnen zusammen mit Grundwasserzuflüssen beruhen. Die Hauptarbeit, welche zu den besprochenen Formen führte, erfolgte zweifellos im Hochglazial. Periglaziale Wirkungen können an der weiteren Ausarbeitung auch noch später beteiligt gewesen sein. 
Mit diesem Ergebnis wird offensichtlich, daß periglaziale Umgestaltung, die ihren Höhepunkt zweifellos vor dem Hochglazial besitzt, auch noch nach Aufschüttung der Außeren Jungendmoräne möglich ist. Besonders interessant ist dabei, daß diese periglaziale Umgestaltung bei unmittelbar zuvor akkumulierten Schottern erfolgte. Auffallend ist schließlich, daß die asymmetrischen Tälchen in eine Schmelzwasserrinne einmünden, die keine Asymmetrie zeigt. Sie ist scharf eingeschnitten und besitzt deutliche Erosionsränder.

Damit wird wieder einmal bewiesen, daß asymmetrische periglaziale Bildungen am schönsten in kleinen Hohlformen auftreten, welche wohl nur mäßige Wassermengen abführten (BüDEL 1944). Die großen Abflußrinnen der Schmelzwässer dagegen wurden schnell und steil durch die Verlegung der oberen Erosionsbasis und die dabei meist trompetentalförmig erfolgende Zerschneidung (C. Troll 1924) von den großen Wassermassen ausgewaschen und eingetieft. Trotz des gleichen Klimas zeigen die Täler auf Grund des unterschiedlich reagierenden Indikators Wasser verschiedene Ausgestaltung.

Bei Begehungen im ganzen Alpenvorland konnte Verf. auch einige der übrigen von RATHJENS (1952) beschriebenen Formen aufsuchen. Die Entstehung dürfte in vielen Fällen ähnlich dem beschriebenen Beispiel erfolgt sein. Allerdings treten dabei vom Lech- bis zum Salzachvorlandgletscher neben den großen Formen der asymmetrischen Täler auch noch kleine Täler auf, die oft nur 200-300 m Länge besitzen (s. Abb. 2). Ob diese allerdings immer mit eiszeitlichen Erscheinungen zusammenhängen, erscheint fraglich. Eine anthropogen bedingte Anlage und eventuelle Grundwassereinwirkungen scheinen nicht unmöglich. Trotz der nunmehr andersartigen Zeitstellung der asymmetrischen Täler beweisen jedoch die von RATHJENS (1952) beschriebenen Formen, daß selbst morphologisch noch manche Feinheiten im Gebiet würmeiszeitlicher Ablagerungen zu erarbeiten sind.

Gleichzeitig mit obigen Untersuchungen des Verfassers, aber völlig unabhängig, bearbeitete Prof. I. Schaefer eine Karte des Isarvorlandgletschers. Dabei stellte er, wie erst eine private Aussprache auf der Tagung der Deuqua in Nürnberg 1962 ergab, ebenfalls fest, daß die asymmetrischen Tälchen, wie vorstehend geschildert, mit Schmelzwasserrinnen in Verbindung stehen, also zweifellos älter sein müssen, als zuvor angenommen.

\section{VI. Überblick über die Akkumulationsphasen seit dem Spätglazial (R. GERMaN)}

Die vorstehend angeführten neuen Beispiele junger Akkumulation im nördlichen Alpenvorland reihen sich recht gut in die bisher bekannten ein und erweitern diese. BRUNNACKER (1960) hat darüber eine ausführliche Zusammenstellung für ganz Bayern vorgelegt. Hier sollen nur die neuen Ergebnisse tabellarisch zusammengestellt und mit den bisher bekannten verglichen werden. Einige weitere Beispiele aus der Literatur sind beigefügt.

Die „zeitweilige Aufschotterungstendenz im Postglazial“ (BrunNacker 1960, S. 94) wird durch die neuen Funde zweifellos unterstrichen.

Der Nachweis der Jüngeren Tundren-Zeit in Krumbach ist besonders bemerkenswert. Dadurch wird gezeigt, daß nicht etwa nur in glazifluvialen Rinnen eine jüngere Akkumulation stattfindet (diese könnte z. B. als ein immer noch wirkender Ausgleichsvorgang der durch die hochglaziale Akkumulation bedingten Gefällstörungen angesprochen werden), sondern daß dies auch in periglazialen Rinnen möglich ist. Außerdem wird offenbar, $\mathrm{da} ß$ die Arbeit des fließenden Wassers, insbesondere die Akkumulationstendenz in den Tälern des nicht vereisten Vorlandes, keineswegs mit dem Hochglazial beendet war. Vielmehr hat die Akkumulation bis in die Zeit der Wiederbewaldung ganz stattliche Beträge erreicht. Offenbar fand auf der damals noch unbefestigten Erdoberfläche, welche noch nicht oder nur von wenigen Pflanzenwurzeln zusammengehalten wurde, doch noch Abspülung statt. Die lockeren Sande der Molasse werden dem abspülenden Niederschlag allerdings auch keinen großen Widerstand entgegengebracht haben. Schließlich ist bei der jungen und 
$\mathrm{T}$ a belle

\begin{tabular}{|c|c|c|c|c|}
\hline $\begin{array}{c}\text { Jahre vor } \\
\text { heute }\end{array}$ & & $\begin{array}{c}\text { Stufe } \\
\text { BrunNACKER } \\
(1960)\end{array}$ & $\begin{array}{l}\text { Pollenzone } \\
\text { Firbas }\end{array}$ & Beispiele junger Akkumulation \\
\hline 0 & & VII ? & $\mathrm{X}$ & \\
\hline 1000 & - & VI ? & 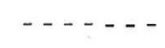 & 1850 J.v.h. Illerschwemmkegel (GraUL \& Gro- \\
\hline 2000 & - & V & IX & $\begin{array}{l}\text { SCHOPF 1952, Groschopf 1961). } \\
\text { Auemergeleindeckung (BRUNNACKER 1960). }\end{array}$ \\
\hline 3000 & - & & VIII & 3140 J.v.h. Rot b. Gaildorf (EISEnHUt 1962). \\
\hline 4000 & - & IV & $\ldots \ldots$ & $\begin{array}{l}4280 \text { J.v.h. Pulling (BrunNaCKEr 1960); Krum- } \\
\text { bach Schicht } 7 ?=\text { Agathazell mächtigste Lage? }\end{array}$ \\
\hline 5000 & - & & VII & \\
\hline 6000 & - & & $-\ldots .$. & \\
\hline 7000 & - & & $\begin{array}{l}\text { VI } \\
-\ldots . . .\end{array}$ & $\begin{array}{l}6650 \text { J.v.h. Traunstein, Bannwaldsee obere Ton- } \\
\text { lage, Agathazell mittlere Tonlage. }\end{array}$ \\
\hline 8000 & - & III & $\begin{array}{l}\mathrm{V} \\
-\ldots . .-\end{array}$ & $\begin{array}{l}\text { Bannwaldsee untere Schicht }=\text { Agathazell untere } \\
\text { Tonlage. }\end{array}$ \\
\hline 10000 & - & II & IV & $\begin{array}{l}\text { Beginn der Tuffbildung (Groschopf 1961). } \\
\text { Basistorf (GrosChOPF 1961). }\end{array}$ \\
\hline 11000 & - & & $\begin{array}{l}\text { III } \\
\text { JTZZ }\end{array}$ & 1045,0 J.v.h. Krumbach Schicht $8=$ ?Kolbermoor? \\
\hline
\end{tabular}

mächtigen Verschüttung periglazialer Täler zu berücksichtigen, daß durch fortgesetzte Umlagerung der Niederterrassenschotter die Mündungen der Periglazialtäler mehr oder weniger verbaut wurden, so daß in deren Mündungsgebieten ebenfalls akkumuliert werden mußte.

Akkumulation aus dem unvergletscherten Gebiet nach dem Hochstand einer Vereisung ist aber auch schon anderweitig nachgewiesen (H. GrauL 1953).

Stellt die zeitliche Fixierung der spätglazialen Probe Krumbach durch die Radiokarbonmethode eine willkommene Bestätigung einer zeitlich an anderer Stelle bekannten Erscheinung, aber in neuem interessantem Zusammenhang dar, so sind wir bei den postglazialen Proben in der Lage, die Stufen III und IV (BRunnacker 1960) gegenüber den bisherigen Angaben genauer zu fassen und teilweise zu ergänzen. Der beim Rotsarimoos zunächst vielleicht zu vermutende lokale Zusammenhang mit dem Schwemmkegel des Halblech (Pendeln auf dem Schwemmkegel) wird durch die gleichzeitig erfolgten Einschwemmungen in Agathazell unwahrscheinlich. Außerdem weisen die klimatischen Überlegungen von P. Firzer (s. Abschn. V a), die aus den verschiedenen Pollendiagrammen zu entnehmen sind, auf regionalen Zusammenhang hin. Schließlich haben wir hiermit aber auch eine Möglichkeit, die Stufe III von BrunNacker (1960), deren Zeitstellung bisher noch nicht ganz sicher war, etwas genauer zu fixieren. Pollenanalytisch gehört sie nach P. Filzer (s. o.) ans Ende der Pollenzone IV (Präboreal). Wichtig ist in diesem Zusammenhang, daß sich die aus dem Pollendiagramm erschlossene Klimaänderung nicht erst plötz- 
lich in den tonigen Sedimenten zeigt (Abb. 1), sondern sich schon vorher andeutet. Das bedeutet, daß nicht etwa das Sediment eine unterschiedliche Erhaltung der Pollen bedingte.

Wenn hier die Einschwemmungen am Ende der Pollenzone IV in die Zeit der Stufe III von BrunNaCKER (1960) eingeordnet werden, so geschieht dies mit Vorbehalt. Die nächstjüngere Probenserie in Pollenzone VI (Atlantikum; siehe Tabelle S. 119), aus welcher der mit Hilfe der Radiokarbonmethode ermittelte Wert $6650 \mathrm{~J}$.v.h. aus Traunstein stammt, konnte von BrunNaCKer in Bayern noch nicht nachgewiesen werden. Vielleicht gehört seine Stufe III oder ein Teil davon in diese jüngere Zeit; vielleicht blieben die Zeitansätze unklar, weil jene Ablagerungen möglicherweise aus zwei verschiedenen Zeiten stammten. $\mathrm{Da}$ zwischen der unteren Einschwemmung in das Profil Rotsarimoos und der oberen (diese ist nach den Ergebnissen der Pollenanalyse etwa zeitgleich mit Agathazell und Traunstein) die ganze Pollenzone V (Boreal) liegt, können beide Erscheinungen schlecht in eine gemeinsame Akkumulationsstufe eingeordnet werden. Das gleichzeitige Vorkommen an drei verschiedenen Stellen des Alpenvorlandes rechtfertigt es wohl, hier eine neue, bisher noch nicht nachgewiesene Akkumulationsphase auszuscheiden.

Die Baumstammlage in $200 \mathrm{~cm}$ Tiefe des Profils Krumbach (s. Profil S. 110), welche auf Grund der Pollenanalyse wohl ins Subboreal, P.Z. VIII, gehört, dürfte mit der Stufe IV (BrunNaCKer 1960) zu parallelisieren sein. Die pollenanalytisch nicht auszuschließende Möglichkeit, daß die Schicht (7) bei Krumbach jünger ist, wird dadurch gering, daß über ihr nochmals eine Baumstammlage (eventuell vom gleichen Alter wie diejenige des Illergletschers) und eine Torfschicht folgen. Eine Radiokarbonbestimmung der Hölzer in den jungen Schichten wäre daher wünschenswert.

Für die Zeitstellung der Stufe IV besitzt BrunNacker (1960) durch den Cl4-Wert von Pulling eine genaue Marke: 4280 J.v.h. Diese Akkumulationszeit fällt ins Subboreal. Aus dieser Zeit hat aber jüngst EISENHUT (1962 S. 3) ebenfalls Akkumulation aus dem württembergischen Stufenland im Remstal nachgewiesen, allerdings mit einem $\mathrm{C}^{14}$-Wert von 3140 J.v.h. (Hv 160: 1180 J.v.Chr.). Es erhebt sich daher die Frage: Hat die Akkumulation im Subboreal rund 1000 Jahre gedauert, oder wurde sie durch untergeordnete Klimaoszillationen unterbrochen, oder liegen aus dieser Zeit mehrere selbständige Aufschüttungen vor?

$\mathrm{Da}$ der Wert aus Pulling an einer Probe von der Basis der Stufe IV stammt, ist eine der beiden ersten Möglichkeiten nicht auszuschließen. Da aber auch die Probe bei ErsenhuT (1962) im unteren Teil einer Schicht vorkommt, muß die dritte Möglichkeit ebenfalls erwogen werden. Auf die Schwierigkeiten bei der klimatischen Ausdeutung der Geländebefunde der Stufen IV und V hat ja schon Brunnacker (1960, S. 138/139) hingewiesen.

In einer Ubersicht über "Neuere flußmorphologische Forschung in Deutschland“ hat unlängst H. BREMER (1961) auf die gegenüber den übrigen Teilen Mitteleuropas abweichende Sedimentationsabfolge der Voralpenflüsse hingewiesen. Dabei wird als Ursache die andere Entwicklungsgeschichte dieser Flüsse im Postglazial vermutet. Mehrere Faktoren können dafür in Frage kommen. Wenn aber in Gebieten des Alpenvorlandes mit relativ geringer Flußgeschwindigkeit kaum Auelehme vorkommen, darf auf die naheliegende Ursache der Gesteinszusammensetzung des Liefergebiets doch wohl nicht verzichtet werden. Selbstverständlich haben diese Nebenflüsse der Donau - verglichen mit denen Mittel- und Norddeutschlands - meist größere Geschwindigkeit. Dadurch wird aber schließlich wieder größere Energie für den Sandtransport (und nicht nur Lehmtransport) ermöglicht. Die Molasse und die Schotterfelder bestehen zum großen Teil aus Sand. Demgegenüber besitzen Ton und Lehm dort ganz geringe Verbreitung. Die (mergelige) Grundmoräne nimmt bei Durchmusterung nach modernen sedimentologischen Methoden immer geringere Flächen ein (GERMan 1962).

Die Bedeutung des geologischen Substrats darf wohl selbst bei gleichartiger klimatischer 
Entwicklung nicht vernachlässigt werden. (Im vorliegenden Fall sind zum Substrat auch Verwitterungsdecken zu rechnen).

In der Zukunft muß besonders die Zeitdauer der Stufen genauer gefaßt werden. Da der Vorgang der Erosion, wie derjenige der damit zusammenhängenden Akkumulation talab wandert, muß mit örtlichen Unterschieden der Ablagerungszeit gerechnet werden. Jedoch sollen nach H. BREMER (1960, S. 294) gestörte Gleichgewichtsverhältnisse schnell ausgeglichen werden.

Obwohl wir bei der Untersuchung geologischer Ablagerungen dazu neigen, einheitlich aussehende Schichten einer bestimmten Zeit zuzuordnen, darf eine subtile Untersuchung und eventuelle Unterteilung nicht vernachlässigt werden. Selbst die Herausarbeitung einer Serie von 8 holozänen Akkumulationen darf uns nicht darüber hinwegtäuschen, daß noch weitere offene Probleme vorliegen. Durch möglichst genaue zeitliche Erfassung der Anfangs- und Endzeiten bei den jüngsten Phasen kann eventuell der Anschluß an die Erforschung der Grenzhorizonte (NILSson 1948, MüNNICH 1960, KubizKi \& MüNNICH 1960), an die atmosphärischen $C^{14}$-Änderungen (WILLIS, T AUBER \& MüNNICH 1960), an die Baumringchronologie und an Klimaänderungen und Gletscherschwankungen erzielt werden. Wie Willis, TAUBER \& Münnich (1960) gezeigt haben, scheint es kürzere und längere Phasen der $\mathrm{C}^{14}$-Schwankung in der Erdatmosphäre gegeben zu haben. Weitere Verknüpfung einwandfrei datierten Tatsachenmaterials könnte zweifellos interessante Zusammenhänge aufzeigen.

Die genaue Kenntnis spätglazialer und holozäner Flußaufschüttungen ist aber auch grundsätzlich wichtig für die Ausdeutung von Interglazialprofilen gleicher Entstehungsart.

P.S. In einer nach Abschluß des Manuskripts erschienenen Veröffentlichung (Jh. Ver. f. vaterl. Naturk. Württ. 118/119, 201-207, Stuttgart 1964) reproduziert H. SCHMEIDL ein Pollendiagramm vom Agathazeller Moor, das in deutlicher Weise die Existenz der oben (V. a) erwähnten Depression der Haselkurve an der Basis einer in Bruchtorf eingelagerten Tonschicht zeigt und damit den Befund von PAUL \& RuOFF (1932) bestätigt. Dasselbe zeigen zwei weitere von mir (FILZER) in den letzten Monaten untersuchte Profile vom Hopfensee nordwestlich von Füssen.

\section{Literaturverzeichnis}

BECKER, E.: Ergebnisse neuer Untersuchungen des Spätwürms im nördlichen Oberrheintal. - Vortrag, gehalten auf der 11. Tagung der Deuqua am 22. Sept. 1962 in Nürnberg.

BERTSCH, A.: Über einen Fund vom allerödzeitlichen Laacher Bimstuff im westlichen Bodenseegebiet und seine Zuordnung zur Vegetationsentwicklung. - Die Naturwissensch. 47, 167, Berlin 1960. - - Untersuchungen zur spätglazialen Vegetationsgeschichte Südwestdeutschlands. Flora 151, 243-280, Jena 1961.

Bremer, H.: Neuere flußmorphologische Forschungen in Deutschland und ausgewählte Probleme der Flußmorphologie deutscher Ströme. - Ber. z. dtsch. Landsk. 25, 283-299, Bad Godesberg 1960.

BRUNNACKER, K.: Die bodenkundlichen Verhältnisse der würmeiszeitlichen Schotterfluren im Illergebiet. - Geolog. Bavarica 18, 113-130, München 1953. - - Zur Kenntnis des Spät- und Postglazials in Bayern. - Geol. Bavarica 43, 74-50, München 1960. - - Das Schieferkohlenlager vom Pfefferbichl bei Füssen. - Jber. Mitt. oberrh. geol. Ver. 44, 61-83, Stuttgart 1962 .

BüDEL, J.: Die morphologischen Wirkungen des Eiszeitklimas im gletscherfreien Gebiet. - Geol. Rdsch. 34, 482-519, Stuttgart 1944. - - Die Frostschutt-Zone Südost-Spitzbergens. Colloquium Geographicum 6, Bonn 1960.

EIsenhut, E.: Pleistozänablagerungen im Neckareinzugsgebiet. - Jber. Mitt. oberrh. geol. Ver. 44, 1-9, Stuttgart 1962.

Firbas, F.: Die Vegetationsentwicklung des mitteleuropäischen Spätglazials. - Bibl. Botan. 112, 68 S., Stuttgart 1935.

Firbas, F., MüNNICH, K. O. \& WITTKE, W.: C14-Datierungen zur Gliederung der nacheiszeitlichen Waldentwicklung und zum Alter von Rekurrenzflächen im Fichtelgebirge. - Flora 146, 512-520, Jena 1958.

German, R.: Die Würmeiszeit im nördlichen Alpenvorland. - Z. deutsch. geol. Ges. (Vortragsreferat). Im Druck. - - Zur Geologie des Lechvorlandgletschers. - Jber. Mitt. oberrh. geol. Ver. 44, 61-81, Stuttgart 1962. 
GöтtLICH, K.: Beiträge zur Entwicklungsgeschichte der Moore in Oberschwaben. Teil I.: Moore im Bereich der Altmoräne und der Äußeren Jungmoräne. - Jh. Ver. vaterl. Naturk. Württ. 115, 93-174, Stuttgart 1960.

Graul, H.: Über die quartären Geröllfazien im deutschen Alpenvorland. - Geol. Bavarica 19, 266-280, München 1953.

Graul, H. \& Groschopf, P.: Geologische und morphologische Betrachtungen zum Illerschwemmkegel bei Ulm. - Ber. naturf. Ges. Augsburg 5, 3-27, Augsburg 1952.

GronBach, G.: Pollenanalytische Untersuchungen zur Geschichte des Federsees und zur vorgeschichtlichen Besiedlung des Federseeriedes. - „Der Federsee“, 316-355, Verl. Schwäb. Albverein, Stuttgart 1961.

Groschopf, P.: Pollenanalytische Datierung württembergischer Kalktuffe und der postglaziale Klimaablauf. - Jh. geol. Abt. württ. Stat. Landesamt 2, 72-94, Stuttgart 1952. - - Beiträge zurHolozänstratigraphie Südwestdeutschlands nach C14-Bestimmungen. - Jh. geol. Landesamt Baden-Württemberg 4, 137-146, Freiburg 1961.

Kubitzki, K. \& MüNNICH, K. O.: Neue C14-Datierungen zur nacheiszeitlichen Waldgeschichte Nordwestdeutschlands. - Ber. deutsch. botan. Ges. 73, 137-146, 1960.

LANG, G.: Zur späteiszeitlichen Vegetations- und Florengeschichte Südwestdeutschlands. - Flora 139, 243-294, Jena 1952. - - Vegetationsgeschichtliche Untersuchungen der Magdalenienstation an der Schussenquelle. - Veröff. geobot. Inst. Rübel Zürich 37, 129-154, Zürich 1962.

Münnich, K. O.: Die C14-Methode. - Geol. Rdsch. 49, 237-244, Stuttgart 1960.

NiLsson, T.: Versuch einer Anknüpfung der postglazialen Entwicklung des nordwestdeutschen und des niederländischen Flachlandes an die pollenfloristische Zonengliederung Südskandinaviens. - Lunds Universitets Arsskrift., NF. Avd. 2, Bd. 44, Nr. 7, Lund 1948.

PAUL, H. \& Ruoff, S.: Pollenstatistische und stratigraphische Mooruntersuchungen im südlichen Bayern, I und II. - Ber. bayer. botan. Ges. 19, 1-84, und 20, 1-264, 1927, 1932.

Rathjens, C.: Asymmetrische Täler in den Niederterrassen des nördlichen Alpenvorlandes. - Geol. Bavarica 14, 140-150, München 1952. - - Ủber Klima und Formenschatz der Späteiszeit. Geol. Bavarica 19, 186-194, München 1953.

ReICH, Helga: Die Vegetationsentwicklung der Interglaziale von Großweil, Ohlstadt und Pfefferbichl im Bayrischen Alpenvorland. - Flora 140, 386-443, Jena 1953.

v. Sarnthein, R.: Moor- und Seeablagerungen aus den Tiroler Alpen in ihrer waldgeschichtlichen Bedeutung. II. Seen der Nordtiroler Kalkalpen. - Beih. bot. Ztrbl. 60/B, 437-492, Dresden 1940. - - Teil III: Österr. bot. Zeitschr. 95, 1-85, Wien 1948.

SchaeeEr, I.: Die Würmeiszeit im Alpenvorland zwischen Riß und Günz. - Abhandl. d. Naturkunde- u. Tiergartenvereins f. Schwaben e. V. Augsburg, H. 2, Augsburg 1940.

Soergel, W.: Diluviale Frostspalten im Deckschichtenprofil von Ehringsdorf. - Fortschr. d. Geol. u. Paläont. 11, H. 36, 439-460, Berlin 1932.

Schreiner, A.: Die Entstehung des Wurzacher Beckens. - Diss. Tübingen 1950.

Troll, C.: Das Inn- und Chiemseevorland. Mitt. geogr. Ges. München 17, 1-43, München 1924. - Die jungglazialen Schotterfluren im Umkreis der deutschen Alpen. - Forsch. z. deutsch. Landes- u. Volksk. 24, 158-256, Stuttgart 1926.

Welten, M.: Pollenanalytische, stratigraphische und geochronologische Untersuchungen aus dem Faulenseemoos bei Spiez. - Veröff. geobot. Inst. Rübel Zürich 21, 201, Zürich 1944. - Pollenprofil Burgäschisee. - Ber. geobot. Inst. Rübel Zürich f. 1946, 101-111, Zürich 1947.

Willis, E. H., H. TAUBER \& K. O. MÜNNICH: Variations in the atmospheric radiocarbon concentration over the past 1300 years. - Amer. J. Sci., Radiocarbon. Supplement 2, 1-4, Chicago 1960

Manuskr. eingeg. 2. 6. 1964.

Anschrift der Verfasser: Priv.-Doz. Dr. R. German, 74 Tübingen, Geologisches Institut der Universität, Sigwartstraße 10. - Prof. Dr. P. Filzer, 74 Tübingen, Ed.-Haber-Str. 14. 